YCTP-N11-95

June 1995

\title{
Universal Predictions for Statistical Nuclear Correlations
}

\author{
Dimitri Kusnezov由 and David Mitchell \\ Center for Theoretical Physics, Sloan Physics Laboratory, \\ Yale University, New Haven, CT 06520-8120 USA
}

\begin{abstract}
We explore the behavior of collective nuclear excitations under a multi-parameter deformation of the Hamiltonian. The Hamiltonian matrix elements have the form $P\left(\left|H_{i j}\right|\right) \propto 1 / \sqrt{\left|H_{i j}\right|} \exp \left(-\left|H_{i j}\right| / V\right)$, with a parametric correlation of the type $\log \langle H(x) H(y)\rangle \propto-|x-y|$. The studies are done in both the regular and chaotic regimes of the Hamiltonian. Model independent predictions for a wide variety of correlation functions and distributions which depend on wavefunctions and energies are found from parametric random matrix theory and are compared to the nuclear excitations. We find that our universal predictions are observed in the nuclear states. Being a multi-parameter theory, we consider general paths in parameter space and find that universality can be effected by the topology of the parameter space. Specifically, Berry's phase can modify short distance correlations, breaking certain universal predictions.
\end{abstract}

PACS numbers: 21.60.Fw, 24.60.Lz, 21.10.Re

\footnotetext{
${ }^{1} \mathrm{E}$-mail: dimitri@nst.physics.yale.edu
} 


\section{Introduction}

The statistics of nuclear excitations has been explored from the shell model to collective models, with studies ranging from the relation of observed quantum fluctuations to those in random matrix models, to the connection with chaos using classical limits of the Hamiltonian [1]- [5]. The agreement of various spectral properties with random matrix predictions has shown that certain simplifying assumptions can be made concerning fluctuations in nuclei. Once random matrix theory can be justified, certain results follow immediately. These studies of chaos in nuclei stem from attempts to extract a simplified behavior from the complexity of nuclear excitations. In this respect, random matrix theory has provided invaluable assistance in developing simple methods to compute complex behaviors. In the past, aside from the studies of constant random matrices and the relation to chaos, these models have been given a parameter dependence to model correlations in various nuclear systems, from heavy ion collisions [6], high spin physics [7] to large amplitude collective motion [8]. Recently it has been shown that Hamiltonians which have a parametric dependence can exhibit universal behavior 9], that is, there can exist model independent quantities in a given theory, providing the Hamiltonian has certain random matrix properties. In this article we study a wide class of observables and develop universal predictions. We further show that parametric deformations of nuclear Hamiltonians can be readily modeled by a simple translationally invariant parametric random matrix theory, even though the Hamiltonian does not apriori look like a random matrix. We further justify the use of parametric random matrix theory for collective nuclear excitations.

\section{Collective Nuclear States}

We have chosen to model collective nuclear excitations in the framework of the Interacting Boson Model (IBM) 10] for several reasons. One of our main objectives is to explore and categorize types of model independent predictions that exist in parametric quantum theories which exhibit classical chaos. The IBM is ideally suited for this since the classical limit has been extensively studied in recent years using coherent states [11], and the complete chaotic behavior is now known for every value of the parameters [12]. Hence we can easily choose parametric variations in regions of strong or weak chaos, or in regular regimes of the parameter space. An additional advantage is that we can solve the quantum problem exactly. One might argue that collective states form only a subset of the real spectrum as the excitation energy increases, so that the use of the IBM is not reasonable. This is not crucial, however, since the IBM provides a solvable theory with known spectral properties, which can be compared to those of the Gaussian Orthogonal Ensemble (GOE) throughout its parameter range. Certainly a more realistic description of the spectrum would embody the same features. For example, when broken pair states are added to the IBM model space, the spectrum becomes more GOE, as the interactions in 
the Hamiltonian become more complicated[13]. This is certainly the case as one attempts to construct more realistic Hamiltonians. And as we are showing how model independent quantities emerge, the model we use is really not so important. Hence we use a simple form of the IBM Hamiltonian, known as the consistent-Q form:

$$
\hat{H}=E_{0}+c_{1} \hat{n}_{d}+c_{2} \hat{\mathbf{Q}}^{\chi} \cdot \hat{\mathbf{Q}}^{\chi}+c_{3} \hat{\mathbf{L}} \cdot \hat{\mathbf{L}}
$$

where

$$
\hat{n}_{d}=d^{\dagger} \cdot \tilde{d}, \quad \hat{L}_{\mu}=\sqrt{10}\left[d^{\dagger} \times \tilde{d}\right]_{\mu}^{(1)}, \quad \hat{Q}_{\mu}^{\chi}=d_{\mu}^{\dagger} s+s^{\dagger} \tilde{d}_{\mu}+\chi\left[d^{\dagger} \times \tilde{d}\right]_{\mu}^{(2)} .
$$

The parameters $c_{i}$ are defined by $c_{1}=\eta / 4$ and $c_{2}=(1-\eta) / 4 N_{b}$, where $N_{b}$ is the number of bosons. Since the Hamiltonian is diagonalized in a basis of fixed angular momentum $L$, the constant $c_{3}$ does not play any role, and is hence omitted. Except when stated otherwise, we will use $N_{b}=25$, which will give optimal statistics for the quantities we consider. The resulting dimensions for $J^{\pi}=0^{+}, 2^{+}, 4^{+}, 10^{+}$states are $65,117,165,211$. In this parameterization, one has the following limits: (a) $\eta=1$ corresponds to vibrational

or $U(5)$ nuclei, (b) $\eta=0$ and $\chi=-\sqrt{7} / 2$ corresponds to rotational or $S U(3)$ nuclei, and (c) $\eta=\chi=0$ describes $\gamma$-soft or $O(6)$ nuclei.

The interpretation of the Hamiltonian in terms of shape variables $\beta$ and $\gamma$ is possible using coherent states, in the large $N$ limit of $H$. The energy surfaces for the Hamiltonian in Eq. (1) is [11]

$$
\mathcal{E}(\beta, \gamma ; \eta, \chi)=\beta^{2} \frac{4-3 \eta}{2}+\beta^{4}(1-\eta)\left(\frac{\chi^{2}}{14}-1\right)+\beta^{3} \cos 3 \gamma \sqrt{1-\frac{\beta^{2}}{2}}(1-\eta) \frac{2 \chi}{\sqrt{7}}
$$

For a particular value of $\eta$ and $\chi$, the energy $\mathcal{E}$ can be minimized to determine the quantities $\beta$ and $\gamma . \beta$ and $\gamma$ in turn define a deformed nuclear mean field. This can be made explicit by re-expressing the Hamiltonian in terms of excitations in a deformed mean field using boson condensate techniques[14]. This allows the interpretation of correlations of observables at different values of $\eta$ and $\chi$ in terms of the shape variable $\beta$ and $\gamma$. Correlations in observables at different values of the parameters are then precisely the correlations between properties of the nucleus in the presence of different mean field configurations. We will consider the behavior of the properties of the Hamiltonian under very general parametric deformation $z=z(\eta, \chi)$. For paths which lie entirely within the chaotic regime of the parameter space, the universal predictions we explore are path independent (up to effects due to Berry's phase which we explore in Sec. 5); correlations in a nucleus changing from rotational to vibrational or vibrational to $\gamma$-soft are the same when properly interpreted.

\subsection{Distributions and Correlations of Nuclear Matrix Elements}

One of the results presented in this article is that parametric nuclear Hamiltonians can be modelled by correlated, parametric gaussian random matrices. Recall that a 
gaussian random matrix has a distribution of matrix elements of the gaussian form $P\left(H_{i j}\right) \propto \exp \left(-H_{i j}^{2} / 2 \gamma\left(1+\delta_{i j}\right)\right)$, where $\gamma$ a constant related to the level density. To implement random matrix theory does not imply that the actual nuclear Hamiltonian (1) have gaussian matrix elements. We note that the distributions of matrix elements of the interacting boson model Hamiltonian are not gaussian. At any given value of $(\eta, \chi)$, we find the distribution of matrix elements obeys roughly 15

$$
P_{i b m}\left(\left|H_{i j}\right|\right) \propto \frac{1}{\sqrt{\left|H_{i j}\right|}} e^{-\left|H_{i j}\right| / V}
$$

where the strength $V$ depends on whether one is in a chaotic or regular regime. Typical results are shown in Fig. 1 for both regular (crosses) and chaotic (boxes) choices of the parameters, together with the behavior (4) (solid curves). In the chaotic parameter regimes of the model, $V$ is of order unity, while in the regular regions, it is much smaller. But both regular and chaotic regimes display the same functional form of the distribution, suggesting that the functional form is due to the structure of the Hamiltonian, rather than to the presence of chaos. Similar distribution functions have been seen in parity non-conservation studies of the compound nucleus 15.

Another quantity of interest is the autocorrelation function $F_{i b m}$ computed from the IBM Hamiltonian:

$$
F_{i b m}\left(z-z^{\prime}\right) \equiv\left\langle H(z) H\left(z^{\prime}\right)\right\rangle=\left\langle\frac{1}{N(N-1)} \sum_{i<j} H_{i j}(z) H_{i j}\left(z^{\prime}\right)\right\rangle_{z-z^{\prime}}
$$

The brackets $\langle\cdots\rangle_{z-z^{\prime}}$ represent the averaging over a trajectory in parameter space $z(\eta, \chi)$, which remains either in a chaotic or in a regular region, keeping the difference $z-z^{\prime}$ fixed. The results for the short distance behavior of the measured function $F_{i b m}$ are shown in Fig. 2, and are found to behave generically as

$$
F_{i b m}(z) \sim e^{-\gamma|z|} \sim 1-\gamma|z|+\cdots
$$

in both regular and chaotic regions. Again, the measured value of $F_{i b m}$ is not a good measure of the underlying chaos. If the averaging in (5) is restricted to a submatrix $N_{1} \leq i, j \leq N_{2}$, there is no difference in the function $F_{i b m}$. One observation is that the apparent decorrelation, seen in the slow decay of $F_{i b m}$, is misleading. The actual decorrelation is much more rapid (when model specific dependencies are removed), as we will see below when we compute properties of wavefunctions and eigenvalues. Hence using Eq. (5) as actual input into a random matrix theory (e.g. into Eq. (16) below) is not meaningful.

\subsection{Unfolded Parametric Energies}

To study the statistical fluctuations of the nuclear energy levels, $E_{n}(z)$, where $z=z(\eta, \chi)$ is a general path in configuration space, we must separate out the average behavior of 
the energies which cannot be described by random matrix theory. This is done with the staircase function [1]

$$
N(E ; z)=\operatorname{Tr} \theta(E-\hat{H})=\sum_{n} \theta\left[E-E_{n}(z)\right]
$$

This function is computed along various paths in parameter space. On each path, 100200 values of $z$ are taken, and the eigenvalues $\left\{E_{n}(z)\right\}$ determined. From this data, a polynomial fit is made to the staircase function using:

$$
N(E ; z)=\sum_{i=0}^{k} \sum_{j=0}^{6} C_{i j} z^{i} E^{j}
$$

In the chaotic regions of parameter space, $k=2$ is sufficient, whereas higher values are needed is less chaotic regions. Once the coefficients $C_{i j}$ are determined, the unfolded energies are defined by

$$
\widetilde{E}_{n}(z)=N\left(E_{n} ; z\right)
$$

which results in a spectrum with a constant average level spacing. The degree of chaos in the energies can be measured through the Brody distribution of the level spacings $s=\widetilde{E}_{n}(z)-\widetilde{E}_{n-1}(z)[16]$ :

$$
P(s)=A s^{\omega} e^{-\alpha s^{\omega+1}}, \quad A=(1+\omega) \alpha, \quad \alpha=\left[\Gamma\left(\frac{2+\omega}{1+\omega}\right)\right]^{1+\omega} .
$$

When $\omega=1$, the distribution of level spacings is GOE, while for $\omega=0$, it is Poisson. In Fig. 3, we show the original (top) and unfolded (middle) parametric energies of the Hamiltonian for $\eta=0$ and $\chi$ as shown. The degree of 'chaos', measured in terms of the Brody parameter $\omega$, is shown in the bottom of the figure for this particular path.

For purposes of contrast, the parametric levels (not unfolded) in two regular regions are shown in Fig. 4. The right figure corresponds to the (regular) transition from $U(5)$ to $O(6)$, while the left corresponds to a path through the 'valley of regularity' recently studied [12] (the two kinks in the parametric energies are artificial and only reflect the fact that the parameter path took a turn). In the figure, the average Brody parameters $\bar{\omega}$ are 0.25 and 0.23 , respectively, with fluctuations up to $\omega=1$. In the following studies, we will consider regular and chaotic regions of the parameter space. As chaos is a classical notion and we study quantum statistics here, let us be precise. The chaotic regions are general paths in parameter space $z(\eta, \chi)$ which stay in the areas of chaos in the classical limit of the Hamiltonian, as discussed in Ref. [12]. The chaotic paths studied here are largely:

$$
z(\eta, \chi)=\left\{\begin{array}{l}
\eta=0, \quad \chi \in[-0.9,-0.4] \\
\eta=0.1, \quad \chi \in[-0.9,-0.4] \\
\chi=-0.66, \quad \eta \in[0.0,0.5]
\end{array}\right.
$$

Similarly, the regular regions we study are:

$$
z(\eta, \chi)=\left\{\begin{array}{lll}
\chi=-\frac{\sqrt{7}}{4} \eta & \eta \in[0,1] & O(6)-U(5) \\
\chi=-0.661, & \eta \in[0.5,1.0] &
\end{array}\right.
$$


Also included in the regular region is the path through the valley of regularity from $S U(3)$ to $U(5)$, as shown in the left half of Fig. 4. The first path in Eq. (12) corresponds to the right half of Fig. 4, is not the direct path between $U(5)$ and $O(6)$ which would be $\chi=0$, $\eta \in[0,1]$, and is entirely integrable. Rather we have chosen one that passes through a weakly chaotic regime. Hence, results in the following sections referred to as 'regular' are quantities which are averaged in the highest $\omega$ regions of these regular areas, such as $z \in[0.5,0.8]$ in Fig. 4 .

\section{Correlated Random Matrix Ensembles}

In order to see what types of model independent quantities emerge from the IBM, we must construct a random matrix model which has an equivalent parametric dependence. There is no unique method to realize such an ensemble, for instance one might take:

$$
\begin{aligned}
H^{\prime}(z) & =H_{1}+z H_{2} \\
H^{\prime \prime}(z) & =\sin z H_{1}+\cos z H_{2} \\
H^{\prime \prime \prime}(z) & =\int d y f(z-y) V(y), \quad \overline{V(z) V(y)}=\delta(z-y) .
\end{aligned}
$$

Here $H_{1}, H_{2}$ are constant $N \times N$ gaussian random matrices and $V_{i j}(y)$ is gaussian white noise for each $i, j$ and $y$. Each of these is a viable random matrix theory, however the stochastic integral of Eq. (15), introduced by Wilkinson [17], provides a more general framework and includes a broader class of processes[18]. One additional difference between $H^{\prime}$ and $H^{\prime \prime}, H^{\prime \prime \prime}$ is that the former is not a translationally invariant theory. While translational invariance is not important to the results we derive here, its presence simplifies our constructions. We will only focus on constructions of the type (14)-(15) here.

The gaussian random matrix Hamiltonians are characterized by their first and second cumulants:

$$
\begin{aligned}
\overline{H_{i j}(z)} & =0 \\
\overline{H_{i j}(z) H_{k l}\left(z^{\prime}\right)} & =\frac{a^{2}}{2 \nu} F\left(z-z^{\prime}\right) g_{i j, k l}^{(\nu)},
\end{aligned}
$$

where $g_{i j, k l}^{(\nu=1)}=\delta_{i k} \delta_{j l}+\delta_{i l} \delta_{j k}, g_{i j, k l}^{(\nu=2)}=2 \delta_{i l} \delta_{j k}$, and a determines the average level spacing $\Delta$ through the relation $a / \Delta=\sqrt{2 N} / \pi$. Here $\nu=1$ (GOE) corresponds to real-symmetric matrices, or equivalently, to a system with time reversal symmetry, and $\nu=2$ (GUE) to complex hermitian matrices, or broken time reversal symmetry. From the definitions of $H(z)$, it is clear that $H(z)$ is $\operatorname{GOE}(\nu=1)$ or GUE $(\nu=2)$ for any $z$.

In contrast to previous studies of chaos in nuclei, which deal with constant random matrices, we can introduce a measure for the parametric ensemble rather easily 18]:

$$
P[H(z)] \propto \exp \left\{-\frac{\nu}{2 a^{2}} \int d z d z^{\prime} \operatorname{Tr}\left[H(z) K\left(z-z^{\prime}\right) H\left(z^{\prime}\right)\right]\right\}
$$


where the measure $D[H(z)] \equiv \prod_{z} d H(z)$ is a product over the continuous variable $z$ of the corresponding gaussian ensemble measure $d H(z)$. Here $K_{i j}(z)$ can be viewed in general as a banded matrix of bandwidth $\sigma$, connecting states $i$ and $j$ with $|i-j| \leq \sigma$. As we do not consider banded parametric matrices here, we will take $K_{i j}(z)=\delta_{i j} K(z)$, resulting in the measure:

$$
P[H(z)] \propto \exp \left\{-\frac{\nu}{2 a^{2}} \int d z d z^{\prime} K\left(z-z^{\prime}\right) \operatorname{Tr}\left[H(z) H\left(z^{\prime}\right)\right]\right\},
$$

These gaussian integrals are easily done to establish Eq. (16), providing $F$ is the inverse of $K$,

$$
\int d z^{\prime} K\left(z-z^{\prime}\right) F\left(z^{\prime}-z^{\prime \prime}\right)=\delta\left(z-z^{\prime \prime}\right) .
$$

The stochastic integral (15) provides a direct method for constructing $H(z)$ with a desired $F(z)$. That is, we can choose $f$ to satisfy

$$
F(z-y)=\int d x f(z-x) f(y-x),
$$

then $H$ is constructed as in (15), and the desired covariance (16) is automatically satisfied. It is important to realize that the properties of the random matrix theory here are distinct from the observed properties of the Hamiltonian, both in the measured distributions of matrix elements $P_{i b m}\left(H_{i j}\right)$ and their autocorrelation $F_{i b m}(z)$. The random matrix distribution of matrix elements is gaussian, and $F(z)$ is different from $F_{i b m}$, as we discuss below. In particular, $F(z)$ cannot be exponential as measured. Model independent results can be obtained from our random matrix constructions by a proper scaling of parameters. We discuss two approaches here to this scaling. The first is a general procedure based on Ref. [18], while the second is a more heuristic argument based on the Fokker-Planck equation and the original work of Dyson, and has been pursued in recent works [19, 20, 21].

\subsection{Scaling from Anomalous Diffusion}

It was shown recently that universal (model independent) predictions can be obtained from the above translationally invariant random matrix theories if one introduces a proper scaling [18]. A general approach to do so is to view the parametric dependence of the energies as a diffusion process. Consider first the short distance behavior of the function $F(z)$ :

$$
F(z) \approx 1-c_{\alpha}|z|^{\alpha}+\cdots \quad .
$$

From perturbation theory, one can see that

$$
\begin{aligned}
\delta E_{n}(x) & =E_{n}\left(x^{\prime}\right)-E_{n}(x)=\delta H_{n n}+\sum_{m \neq n} \frac{\left|\delta H_{m n}\right|^{2}}{E_{n}-E_{m}}+\ldots \\
\left|\left\langle\Psi_{n}\left(x^{\prime}\right) \mid \Psi_{n}(x)\right\rangle\right|^{2} & =1-\sum_{m \neq n} \frac{\left|\delta H_{m n}\right|^{2}}{\left(E_{n}-E_{m}\right)^{2}}+\ldots
\end{aligned}
$$


By implementing the ensemble averages defined in (16), and following Dyson 19, one easily finds that:

$$
\overline{\left(\delta E_{n}(z)\right)^{2}} \simeq \frac{4 N c_{\alpha}}{\nu \pi^{2}} \delta z^{\alpha} \equiv D_{\alpha} \delta z^{\alpha}
$$

and similarly $1-\overline{\left|\left\langle\Psi_{n}(z) \mid \Psi_{n}\left(z^{\prime}\right)\right\rangle\right|^{2}} \propto \delta z^{\alpha}$. One can then view the parametric energy levels $\widetilde{E_{n}}(z)$, such as those in Fig. 2 (middle), as evolving diffusively on short distance scales according to Eq. (24). This has been recently contrasted with the anomalous diffusion process of a particle in a chaotic or disordered medium, whose position obeys < $R^{2}(t)>=D t^{\alpha}$, which although the physics is distinct, the formal treatment is similar 18. For our random matrix model, the parameter $z(\eta, \chi)$ plays the role of time. The diffusion constant $D_{\alpha}$ contains both dimensional information $(N)$ and model dependent data $\left(c_{\alpha}\right)$. Hence, by scaling the parameter $z$ by the diffusion constant $D$, all model and dimension dependence is removed. This is done by defining a new scaled parameter

$$
\widetilde{z}=\left[D_{\alpha}\right]^{1 / \alpha} z=\left(\frac{4 N c_{\alpha}}{\nu \pi^{2}}\right)^{1 / \alpha} z .
$$

For the case of $\alpha=2$, we have $D_{2}=C(0)$, where $C(0)$ is the scaling introduced in Ref. [9]. By computing observables in the scaled variable, one obtains model-independent predictions for desired quantities. Physically, $\alpha=2$ in Eq. (21) corresponds to a Hamiltonian with a smooth dependence on the parameter $z$, while $\alpha<2$ corresponds to a theory with fractal parametric dependence, such as a parameter range taken from a Brownian trajectory. The computation of $D_{\alpha}$ is done through the definition (24):

$$
D_{\alpha}=\frac{\overline{\left(\delta E_{n}(z)\right)^{2}}}{\delta z^{\alpha}}, \quad C(0) \equiv D_{2}=\frac{\overline{\left(\partial E_{n}(z)\right)^{2}}}{\partial z^{2}}
$$

In order to model the IBM Hamiltonian using parametric random matrix theory, we must choose a correlator $F(z)$ in Eq. (16) with $\alpha=2$ short distance behavior (see Eq. (21)). Otherwise the parametric dependence of the random matrix energies $E_{n}(z)$ would not be smooth. Hence, if we attempt to incorporate nuclear properties into the random matrix model by substituting the model specific, computed $F_{i b m}(z)$ in Eq. (6) into Eq. (16), we would end up with an energy spectrum $E_{n}(z)$ characterized by $\alpha=1$, resulting in non-smooth, brownian motion type paths for each $E_{n}(z)$. For the IBM, $\alpha=2$ is the proper result. Details can be found in Ref. [18].

\subsection{Scaling and the Fokker-Planck Equation}

A more heuristic argument can also be made with the Fokker-Planck equation. FokkerPlanck methods, first introduced by Dyson [19], have been recently discussed with parametric correlations in mind 20]- 21]. Consider first Dyson's Brownian motion model for random matrices:

$$
\dot{H}_{i j}=-\gamma H_{i j}+f_{i j}(t)
$$


The random force is white noise:

$$
\begin{aligned}
\overline{f_{i j}(t)} & =0 \\
\overline{f_{i j}(t) f_{k l}^{*}\left(t^{\prime}\right)} & =\Gamma g_{i j, k l}^{(\nu)} \delta\left(t-t^{\prime}\right) .
\end{aligned}
$$

and $\gamma$ is a friction coefficient. The equilibrium solution can be found in the long time limit, by direct integration:

$$
H_{i j}(t)=\int^{t} e^{-\gamma|t-\tau|} f_{i j}(\tau) d \tau
$$

which is same type of stochastic formulation as in Eq. (15). It follows then from Eqs. (27,28,29) that:

$$
\left\langle H_{i j}(t) H_{k l}\left(t^{\prime}\right)\right\rangle=\frac{\Gamma}{2 \gamma} g_{i j, k l}^{(\nu)} e^{-\gamma\left|t-t^{\prime}\right|}
$$

This process can be formulated as well in terms of the Fokker-Planck equation for the distribution $P(H, t)$ :

$$
\frac{\partial P}{\partial t}=\frac{\partial}{\partial H_{i j}}\left(\gamma H_{i j} P\right)+\frac{1}{2} g_{i j, j i}^{(\nu)} \Gamma \frac{\partial^{2} P}{\partial H_{i j} \partial H_{i j}^{*}} .
$$

Since we are interested in a stationary process $P(H, t)=P(H)$ for $H(t)$, we can choose the initial distribution to be the equilibrium result $P(H) \propto \exp \left[-\nu \operatorname{Tr} H^{2} / 2 a^{2}\right]$. The equilibrium solution is a solution of the Fokker-Planck equation providing the fluctuation dissipation theorem is satisfied:

$$
g_{i j, j i}^{(\nu)} \Gamma / 2 \gamma=\overline{\left|H_{i j}\right|^{2}}=\frac{a^{2}}{2 \nu} g_{i j, j i}^{(\nu)}
$$

or $a^{2} / \nu=\Gamma / \gamma$, where the last equality follows from our original construction in Eq. (16). Following Dyson's argument that the eigenvalues of $H(t)$ behave as a diffusive coulomb gas, equilibrating (at the microscopic scale) on a timescale of $t \propto 1 /(\gamma N)$, we see that in order to obtain $N$-independent correlations, we must have $\gamma \propto 1 / N$, or $\gamma=\gamma^{\prime} / N$. By equating the second cumulants of $H(t)$ for and Langevin process (30) and for our desired process (16), and implementing the fluctuation-dissipation theorem, we equate

$$
F\left(z-z^{\prime}\right) \leftrightarrow \exp \left(-\frac{\gamma^{\prime}}{N}\left|t-t^{\prime}\right|\right) .
$$

Expanding to leading order in the large $N$ limit, we associate:

$$
1-c|z|^{n} \simeq 1-\frac{\gamma^{\prime}}{N}|t|
$$

As the Langevin result is $N$-independent at the microscopic scale, $N$ independent results will also result if we define a new quantity $\hat{z}=(c / N)^{1 / n} z$. Up to a factor of order unity $\left(4 / \pi^{2} \nu\right)$, this is precisely the same scaling we discussed earlier. Now to leading order,

$$
F \approx 1-\nu \frac{\pi^{2}}{4} \frac{\left|\widetilde{z}-\widetilde{z}^{\prime}\right|^{n}}{N}
$$


There is now no explicit model dependence $c$, and the $N$-dependence of correlation functions will be absent due to the explicit dependence on $N$, which was require to achieve the microscopic equilibrium condition discussed above.

\section{Observables}

In this section we will see that many properties of parametric Hamiltonians have well defined model independent structure. There are two classes of observables we study here, those related to the energies $E_{n}(z)$ and those related to the instantaneous eigenfunctions $\left|\Psi_{n}(z)\right\rangle$. The relation of wavefunctions $\left|\Psi_{n}(z)\right\rangle$ at $z$ to those at $z^{\prime}$ is given by the transformation matrix:

$$
U_{n m}\left(z-z^{\prime}\right)=\left\langle\Psi_{n}(z) \mid \Psi_{m}\left(z^{\prime}\right)\right\rangle
$$

We will see below that correlation functions that depend on $U_{n m}(z)$ and $E_{n}(z)$ have universal predictions which generally agree well with the results of the IBM in the chaotic regions.

The universal predictions for an $\alpha=2$, GOE system are computed here with two different covariances. The first is the simple sum of two uncorrelated GOE matrices

$$
H^{\prime}(z)=H_{1} \cos z+H_{2} \sin z, \quad \overline{H^{\prime}(z) H^{\prime}(y)}=\cos (z-y)=F^{\prime}(z-y) .
$$

The correlator is periodic with $F^{\prime}(z) \approx 1-z^{2} / 2+\cdots$, defining the scaling $z \rightarrow \widetilde{z}=$

$\sqrt{D_{2}} z=(\sqrt{2 N} / \pi) z$ for universal correlations. The second construction is in terms of a stochastic integral, where one integrates over a continuous range of uncorrelated random matrices $V(y)$ :

$$
H^{\prime \prime}(z)=\int d y e^{-(z-y)^{2} / 2} V(y), \quad \overline{H^{\prime \prime}(z) H^{\prime \prime}(y)}=e^{-(z-y)^{2} / 4}=F^{\prime \prime}(z-y)
$$

Here $\widetilde{z}=(\sqrt{N} / \pi) z$. We have computed various correlation functions described below with $N=50-300$. Generally $N=50$ is sufficiently large.

\section{$4.1 \quad \mathrm{C}(\mathrm{z})$}

The slope-slope correlation function of the unfolded parametric energies $\widetilde{E_{n}}(z)$ is defined as 9

$$
C\left(z-z^{\prime}\right)=\left\langle\frac{\partial \widetilde{E}_{i}(z)}{\partial z} \frac{\partial \widetilde{E}_{i}\left(z^{\prime}\right)}{\partial z}\right\rangle_{E, z}
$$

where the averaging is over energy and parameter. In Fig.5, the results for the IBM in the chaotic regions are shown for $J^{\pi}=0^{+}, 2^{+}, 4^{+}, 10^{+}$. The averages are computed by averaging over the middle third of the spectrum and over the trajectory $z$. The two solid lines are the results of the random matrix simulations, Eqs. (37-38), for $N=50$ and 
300. For comparison, a computation in the regular region of the IBM is shown indicating much slower decorrelation. As we will see in all computations here, the typical distance at which quantities decorrelate is $\widetilde{z} \sim 1$, which corresponds to the average separation between level crossings when the energies $\widetilde{E}_{n}$ are plotted as a function of the scaled parameter $\widetilde{z}$. For regular systems, and the apparent level crossings in Fig. 4, this is not the case, and decorrelation happens over a much longer scale. In general the agreement with the universal functions is quite good. The $0^{+}$states have the poorest agreement, which is in part statistical, having the smallest dimension.

\section{$4.2 \quad$ N-Scaling of $\mathrm{C}(0)$}

We have seen that the diffusion constant $C(0)=D_{2}$ scales linearly with $N$, the dimension of the space. This scaling can be tested in the IBM by modifying the boson number $N_{b}$. For $N_{b}=10,15,20,25$ we have dimensions of $J=10^{+}$states of $N=16,56,121,211$. In Fig. 6 we plot $C(0)$ as a function of $N$, and see that this scaling is observed. At low $N_{b}$ (equivalently low $N$ ), the results are not as reliable due to statistics becoming increasingly poor, and the classical phase space becoming increasingly regular 12 .

\subsection{Curvature Distribution $\mathrm{P}(\mathrm{k})$}

The distribution of curvatures of the parametric energies $E_{n}(z)$, or equally $\widetilde{E_{n}}(z)$, have a predicted distribution in the chaotic regime, given by 22]:

$$
P(k)=\frac{c_{\nu}}{\left(1+k^{2}\right)^{(\nu / 2+1)}}, \quad k=\frac{d^{2} \widetilde{E_{n}}}{d z^{2}} \frac{1}{\pi \nu C_{0}} .
$$

Here $c_{\nu}$ is the normalization, $\nu=1(2)$ for $\operatorname{GOE}(\mathrm{GUE})$, and $k$ is the scaled curvature of the parametric energy. This function is compared to our random matrix computation (Eq. (38)) in Fig.7 (a). In Fig. 7 (b), the results for the chaotic region of the IBM (solid histogram) are seen to agree equally well. A similar calculation done in the regular region shows a much more strongly peaked function (dashed, and scaled by $1 / 5$ vertically). This is expected since the regular regions have much fewer level crossings and are hence much flatter (see Fig. 4).

\subsection{Diagonal Wavefunction Decorrelations: $P_{n}\left(\left|U_{n n}(z)\right|^{2}\right)$}

The adiabatic survival probability $\left|\left\langle\Psi_{n}(z) \mid \Psi_{n}(0)\right\rangle\right|^{2}$ measures how rapidly wavefunctions decorrelate. This was shown to be universal recently 18, 23, with a well defined Lorentzian shape:

$$
P_{n}\left(\left|U_{n n}(z)\right|^{2}\right)=\overline{\left|\left\langle\Psi_{n}(z) \mid \Psi_{n}(y)\right\rangle\right|^{2}}=\left(\frac{1}{1+c|\widetilde{z}-\widetilde{y}|^{\alpha}}\right)^{\nu}
$$


where $\nu=1(2)$ corresponds to GOE(GUE) eigenstates, $c$ is a constant, and $\alpha$ is given by the leading order behavior of $F(z)$. For the case at hand, $\nu=1$ and $\alpha=2$. In Fig. 8, comparisons are shown for selected spins, and in chaotic (symbols) and regular (dashed) regions. The regular regions indicate much longer correlations, while the GOE result provides the most rapid statistical decorrelation of states. The two solid lines are the random matrix predictions. There is no equivalent universal prediction for the nonchaotic regimes, and the dashed line is just a representative $P_{n}$ of the IBM in the regular region.

\subsection{Off-Diagonal Wavefunction Decorrelation: $P_{k}\left(\left|U_{n m}(z)\right|^{2}\right)$}

Wilkinson and Walker 21 have used perturbation theory to derive an approximate expression for the distribution of squared off-diagonal matrix elements, $\left|\left\langle\Psi_{n}(z) \mid \Psi_{m}(0)\right\rangle\right|^{2}$, in the limit of $|z| \rightarrow \infty$ and $k=|m-n| \gg 1$. They found that

$$
P_{n m}(z)=\frac{\mu^{2} z^{2}}{\left(E_{n}-E_{m}\right)^{2}+\left(\pi \rho \mu^{2} z^{2}\right)^{2}}, \quad \mu^{2}=\left\langle\left|\frac{\partial H}{\partial z}\right|^{2}\right\rangle_{E_{n} \sim E_{m}, n \neq m}
$$

Here, the energies are not averaged over, $\mu^{2} \sim 1$, and $\rho$ is the average level density. In exploring the behavior of these quantities in the IBM, it is difficult to satisfy the validity conditions for Eq. (42), since we largely study states in the middle portion of the spectrum, and $m \gg n$ is difficult to satisfy. If we use only two states separated by $k=|n-m| \gg 1$, and we do not average over energy, the statistics are very poor. In order to get good statistics, we have examined the equivalent distribution which is averaged over both coordinate $z$ and energy, with $k$ kept fixed. We define this distribution of off-diagonal matrix elements as $(k>0)$ :

$$
P_{k}(\widetilde{z})=\left\langle\left|U_{n m}(z)\right|^{2}\right\rangle=\left\langle\left|\left\langle\Psi_{n}\left(z+z_{o}\right) \mid \Psi_{m}\left(z_{o}\right)\right\rangle\right|^{2}\right\rangle_{E, z_{o}, k=|n-m|}
$$

where the subscript indicates that it is averaged over the trajectory $z(\eta, \chi)$, as well as over energy, with the separation $|n-m|$ held fixed. We use the notation $P_{n m}$ for the quantity which is not energy averaged, and $P_{k}$ for the energy averaged result. In Fig. 9, we compare this function computed in random matrix theory (solid) and in the chaotic regime of the IBM (boxes). In general the agreement is quite good. The random matrix theory result was done using $N=50$, and averaging over the middle third of the spectrum. Hence as $k$ increases, the statistics get worse. The IBM results are for the $J^{\pi}=10^{+}$states, with a dimension of 211, so that the statistics is better. In order to contrast our results with Eq. (42), we have taken a rescaled form, which is not entirely justified, as the scaling by $\mu$ is distinct from $D_{2}=C(0)$. Nevertheless, we plot in Fig. 9, the following rescaled functions (whose regimes of validity are indicated):

$$
\begin{aligned}
P_{k}^{\prime}(\widetilde{z}) & =\frac{\widetilde{z}^{2}}{k^{2}+\widetilde{z}^{4}}, \quad k \gg 1,|z| \gg 1 \\
P_{k}^{\prime \prime}(\widetilde{z}) & =\int_{0}^{\infty} d x \cos (k x) e^{-\widetilde{z}^{2}[1-\exp (-|x|)]}, \quad k \gg 1
\end{aligned}
$$


The function $P_{k}^{\prime}$ (dots in Fig. 9) is an approximation of $P_{k}^{\prime \prime}$ (dot-dashed in Fig. 9) in the limit $|z| \gg 1$, which was derived in Ref. [21]. As the value of $k$ increases, there is better agreement with the exact results form the random matrix simulation and the IBM. We observe that a better overall fit can be found with the function (dashed line if Fig. 9)

$$
P_{k}(\widetilde{z})=\frac{\widetilde{z}^{2}}{k(k-c)+\widetilde{z}^{4}}, \quad c=3 / 4
$$

which trivially converges to the Wilkinson-Walker result in its regime $k \gg 1$, but better describes the results for all $k$. The results for the regular region $\chi=-0.661, \eta \in[0.5,1.0]$, are given by the crosses. Once again, there is no universal result for the regular case. Further, as expected, all three analytic functions (42)-(44) agree in the large $k$ limit.

\subsection{Diagonal Matrix Elements: $P_{\widetilde{z}}\left(U_{n n}(z)\right)$}

The previous results have been averages over various matrix elements. We now show that the actual distributions of matrix elements can also be predicted by universal functions. Consider, for example, the distribution of the matrix elements $U_{n n}(z)=\left\langle\Psi_{n}(z) \mid \Psi_{n}(0)\right\rangle$. These can be seen to be described by a universal function for each $z$. The distribution $P\left(U_{n n}(\widetilde{z})\right)$ is shown in Fig. 10 for $J^{\pi}=10^{+}$states at several values of $\widetilde{z}$. At $z=0$, the distribution is a $\delta$-function centered at 1 . As the separation of the wavefunctions increases, the centroid shifts from 1 to 0 , and the overlap evolves to an asymptotic PorterThomas distribution (an approximate gaussian) at large $z$ :

$$
P_{\widetilde{z}}\left(U_{n n}(z)\right) \rightarrow \begin{cases}\delta\left(U_{n n}-1\right) & \widetilde{z} \rightarrow 0 \\ \exp \left(-N U_{n n}^{2} / 2\right) & \widetilde{z}>1\end{cases}
$$

The large $z$ limit is not universal, as can be seen by the explicit dimensional dependence of the result. The Porter-Thomas result lies beyond the range of universality which is largely $\widetilde{z} \leq 1$, but the precise decorrelation of matrix elements within this range has the model independent form shown in Fig. 10.

\subsection{Off-Diagonal Matrix Elements: $P_{\widetilde{z}}\left(U_{n m}(z)\right)$}

A similar result exists for the distribution of off-diagonal matrix elements $U_{n m}(z)=$ $\left\langle\Psi_{n}(z) \mid \Psi_{m}(0)\right\rangle$. The distribution $P_{\widetilde{z}}\left(U_{n m}(\widetilde{z})\right)$ is shown in Fig. 11 for $J^{\pi}=10^{+}$states at several values of $\widetilde{z}$. At $z=0$, the distribution is also a $\delta$-function, but now centered at $z=0$. As the separation of the wavefunctions increases, the overlaps evolve to an asymptotic Porter-Thomas distribution (an approximate gaussian) at large $z$ :

$$
P_{\widetilde{z}}\left(U_{n m}(z)\right) \rightarrow \begin{cases}\delta\left(U_{n m}\right) & \widetilde{z} \rightarrow 0 \\ \exp \left(-N U_{n m}^{2} / 2\right) & \tilde{z}>1\end{cases}
$$


As before, the large $z$ limit is not universal, as can be seen by the explicit dimensional dependence of the result. The solid curve in Fig. 11 is the Porter-Thomas result.

\subsection{Correlations of Mean Fields}

Each value of $(\eta, \chi)$ corresponds to a deformed mean field characterized by $(\beta, \gamma)$ de-

termined from the minimum of Eq. (3). Because wavefunctions decorrelate on order of $\widetilde{z}=\sqrt{D_{2}} z \sim 1$, the actual correlation length in terms of the parameters $\eta$ and $\chi$ depends on spin, and is given by $z \sim z_{c} \equiv 1 / \sqrt{D_{2}\left(\Delta \beta, \Delta \gamma, J^{\pi}\right)}$. To explore the spin dependence of the correlation length, we compute $z_{c}$ in the IBM for $J^{\pi}=0^{+}, 2^{+}, 4^{+}, 10^{+}$, and find typical values of $z_{c}=0.16,0.14,0.11,0.05$. This has a roughly behavior

$$
z_{c} \sim 1-\gamma J
$$

where $\gamma$ is a constant. How generic such an dependence might be in other nuclear models is unclear, but it does indicate how rapid states of different spin can decorrelate (n.b. the results can be corrected for the dependence of $z_{c}$ on $\sqrt{N}$, but this does not account entirely for the behavior). The equivalent values of $\Delta \beta$ and $\Delta \gamma$, which correspond to statistically decorrelated configurations, depend rather strongly on the parameter region. For example, in our calculations we can obtain a range from $\Delta \beta=0.01$ to 1.3 , for the same correlation length, depending on whether the shape is undergoing a rapid shape phase transition or not in the particular parameter regime. One can only conclude that near a shape phase transition, there can exist strong statistical correlations between very distinct nuclear shapes.

\subsection{More Complicated Operators}

It is clear that one can explore many classes of operators and establish the behavior of model-independent limits of those quantities. For instance in the study of the $E 2$ decay of high-spin states, Aberg[7] has introduced the matrix quantity

$$
T_{i j}=\left|\left\langle\Psi_{i}(J-2) \mid \Psi_{j}(J)\right\rangle\right|^{2}\left(E_{j}(J)-E_{i}(J-2)\right)^{5}
$$

where the parameter $J$ is the angular momentum, and is equivalent to $z$. This matrix can be explored as a function of the correlation length, and has different results in the chaotic limit, depending on the spin dependent scaling $D_{2}$. One can consider other operators as well, and we would like to point out that additional quantities can be constructed using our universal predictions here, together with the analysis of Ref. [21], which discusses how to compute arbitrary correlation functions. 


\section{Multi-parameter Correlations: Topological Effects and Berry's Phase}

While formal studies of parametric correlations have been limited largely to single parameter systems (see Ref. [21] for some exceptions), nuclear deformation is usually described in terms of two or more shape parameters. When two or more parameters are involved, one finds that short distance correlations can be modified by topological effects, due to Berry's phase. That is, the correlation between quantities at $\beta, \gamma$ and $\beta^{\prime}, \gamma^{\prime}$ depend on the path used to connect these points. Generally, for correlation functions which are sensitive to phase information, we will show that interference terms can strongly modify the expected results. We explore the basic ideas here in the case of two parameters.

When a wavefunction undergoes parametric evolution on a closed circuit $C$, it is well known that the wavefunction can pick up a topological phase:

$$
\Psi_{n}(z) \longrightarrow e^{i \gamma(C)} \Psi_{n}(z)
$$

where $C$ represents a loop in parameter space starting and ending at $z$. For real symmetric matrices, such as our GOE ensemble, $\gamma(C)$ is only 0 or $\pi(\bmod 2 \pi)$ [24]. Hence

$$
\Psi_{n}(z+C)= \pm \Psi_{n}(z)
$$

where the sign depends upon the particular eigenstate and the path, and $z+C$ represents the same point $z$ after following the closed loop $C$. Of course, one does not have to follow a closed loop. A similar effect exists if one follows two distinct paths from $z$ to $z^{\prime}$. Then phase differences result in interference. Whether or not paths are in a chaotic or regular regime does not change the flavor of the argument, but in the chaotic regime, more states are likely to pick up a negative phase due to the many avoided level crossings[25].

\subsection{A 2-Parameter Random Matrix Model}

The simplest formulation of a two parameter correlated random matrix ensemble is

$$
H(X, Y)=H_{1} \cos X+H_{2} \sin X+H_{3} \cos Y+H_{4} \sin Y
$$

where the constant random matrices $H_{i}$ are uncorrelated: $\overline{H_{i} H_{j}}=\delta_{i j}$. It follows that

$$
\overline{H(X, Y) H\left(X^{\prime}, Y^{\prime}\right)}=\cos \left(X-X^{\prime}\right)+\cos \left(Y-Y^{\prime}\right) .
$$

Generalizations to arbitrary dimensions have been discussed by Wilkinson 21]. We can now consider parametric excursions in the $(X, Y)$ plane, specifically two paths which

connect $(0,0)$ to $(\Delta X, \Delta Y)$, one of shortest length, and the other a longer path enclosing an area $A$. Because the wavefunctions acquire a Berry's phase around the closed loop, which can be \pm 1 for the GOE case, the area $A$ enclosed can modify expected short distance behaviors. 


\subsection{Correlations in the $\beta-\gamma$ plane}

We can now explore some of the topological effects in our two parameter theory $H(\eta, \chi)$. Consider a rectangular loop $C$ in parameter space which encloses an area $A$. In analogy to scaled parameter $\widetilde{z}$, we define the scaled area of the loop as $\widetilde{A}=C(0) A \sim \widetilde{z}^{2}$. Then an area $\widetilde{A} \sim 1$ is a loop whose sides are approximately the decorrelation length of observables. Such a loop stays within the universal regime for all values of the parameter. In Fig. 12, we plot the distribution of matrix elements $P_{z}\left(U_{n n}\right)$ (see Eq. (45)) for such a loop. Starting from the top of the figure, we have $\widetilde{z}=0$, and the distribution is a delta function. As $\widetilde{z}$ increases, the distribution spreads in accordance with universal predictions (cf. Fig. 10). At the farthest point of the loop, the distribution is given by the middle figure. As the trajectory returns to the initial point, approximately half the eigenfunctions develop a negative topological phase, and at the final point, which is precisely the initial point, the distribution is equally split. All of the results in Fig. 12 are within the universal regime, but one can see that topological effects can destroy the expected behavior discussed in Fig. 10. For smaller loops, the effect is smaller. The approximate behavior is[25]

$$
f=\left\{\begin{array}{cc}
\widetilde{A} & \widetilde{A} \leq 1 \\
\frac{1}{2} & \widetilde{A}>1
\end{array}\right.
$$

Here $f$ is the fraction of the total states which split to -1 , and $\widetilde{A}$ is the enclosed, scaled area. The fraction increases linearly with the area. Because saturation occurs near $\widetilde{A}=$ $1=C(0) \Delta \chi \Delta \eta$, and $C(0) \propto N$, the size of the loop needed to see the maximal effect decreases like $1 / N: \Delta \chi \Delta \eta \sim 1 / N$. Hence we see that universal predictions can be modified in multi-parameter theories due to the topology of the parameter space. It is not sufficient to give only the metric distance in parameter space in order to provide all universal predictions. One must also consider the path taken to get to that metric separation.

So there are several aspects here to consider. Berry's phase effects are independent of the underlying chaos along the chosen path, but depend more upon the nature of the parameter space enclosed by the path. One could imagine a loop in parameter space which is entirely regular, but encloses a chaotic regime. The expression for the fraction of total states $f$ above assumes one is always in a chaotic regime, and the enclosed area is also chaotic.

\section{Conclusions}

In conclusion, we have explored the adiabatic behavior of collective nuclear excitations, and found that under the appropriate scaling of the parameter, correlation functions and distributions of matrix elements behave universally. Hence, if we wish to implement random matrix theory to study a complex nuclear situation, we specify immediately a 
multitude of model independent results related to the wavefunctions and energies. The results here indicate that a new universality exists in nuclei, related to the 'deformation' of the nucleus, which is quite robust. As the random matrix predictions are generic, they should be present in other classes of nuclear states, generated from the shell model, or other models. While we have focused on matrix element distributions and certain correlation functions, it is clear that the scaling provides a general type of approach to compute arbitrary correlation functions. This also establishes that the use of random matrix theory with covariances of the type (16) is quite reasonable.

We would like to thank R. Casten, C. Lewenkopf and V. Zamfir for useful discussions, and M.Wilkinson for providing an advance copy of Ref. [21]. This work was supported by DOE grant DE-FG02-91ER40608. 


\section{References}

[1] See for example, O. Bohigas, in Chaos and Quantum Physics, Eds. M. Giannoni, A. Voros and J. Zinn-Justin, (North-Holland, New York, 1991); O.Bohigas and H.A.Weidenmüller, Ann. Rev. Nucl. Part. Sci. 38 (1988) 421.

[2] M.Horoi, V.Zelevinsky, and B.A.Brown, Phys. Rev. Lett. 74 (1995) 5194; D.C.Meridith, S.E.Koonin, and M.R.Zirnbauer, Phys. Rev. A37 (1988) 3499.

[3] V. Paar and V. Vorkapic, Phys. Lett. B205 (1988) 7.

[4] S. Drozdz, S. Nishizaki, J. Speth and J. Wambach, preprint nucl-th/9407015, (1994).

[5] J. Shriner, Jr., G.E.Mitchell, and T.von Egidy, Z. Phys. A338 (1991) 309; J.M.Espino and J.D.Garrett, Nucl. Phys. A492 (1989) 205.

[6] D.Brink, H.Weidenmuller, and, Phys. Lett. B (1978)

[7] S. Aberg, Lund preprint Lund-MPh-94/08, (1994).

[8] A.Bulgac, G. DoDang and D.Kusnezov, Ann. Phys. (NY) 242 (1995) 1.

[9] A. Szafer and B. L. Altshuler, Phys. Rev. Lett. 70, 587 (1993); B.D. Simons and B.L. Altshuler, Phys. Rev. Lett. 70, 4063 (1993); Phys. Rev. B 48, 5422 (1993).

[10] F. Iachello and A. Arima, The Interacting Boson Model (Cambridge Press, Cambridge, 1987).

[11] J.Ginnocchio and M.W.Kirson, Nucl. Phys. A350 (1980) 31; D. Feng, R. Gilmore and S.R.Deans, Phys. Rev. C23 (1981) 1254.

[12] N. Whelan, Ph.D. thesis, Yale University (1993).

[13] F. Iachello and D.Vrentenar, Phys. Rev. C43 (1991) R945; V.Paar, D. Vorkapic and K.Heyde, Phys. Lett. B211 (1990) 265; H.Wu, D.H.Feng, and M.Vallieres, J. Phys. G16 (1990) L149; Y. Alhassid and D. Vretenar, Phys. Rev. C46 (1992) 1334.

[14] A. Leviatan and M.W.Kirson, Ann. Phys. (NY) 188 (1988) 142.

[15] V.V.Flambaum, A.A.Gribakina, G.F.Gribakin and M.G.Kozlov, Phys. Rev. A50 (1994) 267.

[16] T.Brody, J.Flores, J. French, P.Mello, A.Pandey and S.Wong, Rev. Mod. Phys. 53 (1981) 385.

[17] M.Wilkinson, Phys. Rev. A41 (1990) 4645.

[18] D.Kusnezov and C.Lewenkopf, Yale University preprint YCTP-N2-95, (1995) 
[19] F.J. Dyson, J. Math. Phys. 3, 1191 (1962).

[20] C.W.J.Beenakker, Phys. Rev. Lett. 70 (1993) 4126; Y.Alhassid, D. Kusnezov, and D. Mitchell, Yale University preprint (1994); D. Mitchell and D.Kusnezov, preprint (1995).

[21] M. Wilkinson and P. Walker, J. Phys. A (in press) (1995).

[22] J. Zakrzewski and D.Delande, Phys. Rev. E47 (1993) 1650; F. von Oppen, Phys. Rev. Lett. 73 (1994) 798.

[23] D.Kusnezov and D.Mitchell, Yale University preprint YCTP-N4-95 (1995).

[24] M.Berry and M.Wilkinson, Proc. Roy. Soc. Lond. A392 (1984) 15.

[25] D.Mitchell, in preparation. 


\section{Figures}

Figure 1. Distribution of matrix elements for the IBM in the chaotic region (boxes, $\eta=0, \chi=-0.7)$, and in the regular region (crosses, $\eta=0.85, \chi=-0.661$ ). Both

distributions are compared to the distribution $P(|H|) \propto 1 \sqrt{|H|} \exp (-|H| / V)$, with values of $V=10$ and $V=0.3$, respectively.

Figure 2. Short distance behavior of the measured IBM autocorrelation function $\langle H(z) H(0)\rangle$ in the chaotic region (solid) and in the regular region (dots). The linear behavior at small $z$ suggests $\alpha=1$ in Eqs. (21) and (26), which is not consistent with the observed parametric energies $E_{n}(z)$. Hence $F_{i b m}$ cannot be used as physical input into the random matrix theory through Eq. (16).

Figure 3. Instantaneous eigenstates of the Hamiltonian (1) for the parameter range $\eta=0$ and $\chi$ as shown. (Top) Original energies; (Middle) unfolded eneries; (bottom) Brody parameter along this path, indicating a rather chaotic regime, $\omega=1$ being the GOE limit.

Figure 4. Instantaneous eigenstates of the Hamiltonian (1) for two largely regular regions. (Left) A path from rotational $(S U(3))$ to vibrational $(U(5))$ spectra through the regular region proposed recently[12]. The energies have been scaled by $1 / 2$. (Right) The transition from vibrational $(U(5))$ to $\gamma$-unstable $(O(6))$ choosing a path which is weakly chaotic. The average Brody parameters are $\bar{\omega}=0.23$ and $\bar{\omega}=0.25$, respectively.

Figure 5. Slope-slope autocorrelation function, $C(\widetilde{z})$, for the parametric energies $\widetilde{E}_{n}(\widetilde{z})$ in the chaotic (symbols) and regular (dots) regions. The solid lines are the random matrix predictions using (37) and (38) with $N=300$ and $N=50$, respectively. The $0^{+}$states have the poorest statistics, due in part to the small dimension of the space $(\mathrm{N}=65)$.

Figure 6. Scaling of $D_{2}=C(0)$ with $N$, the dimension of the space. The boson number was varied as $N_{b}=10,15,20,25$, resulting in dimensions $N=16,56,121,211$ for $J^{\pi}=10^{+}$ states. The anticipated scaling behavior, given by Eqs. (25)-(26) with $\alpha=2$, is linear, shown by the solid line. There are deviations at small boson number since the chaos is not as strong there, and the dimensions are small.

Figure 7. (a) Analytic level curvature distribution $P(k)$ (solid) compared to results of a GOE simulation (histogram). (b) Comparison of the analytic distribution to those for $2^{+}$ states in the IBM (solid histogram). The dashed histogram corresponds to $2^{+}$states in the regular regime, and has been scaled vertically by $1 / 5$.

Figure 8. Wavefunction decorrelation function $P_{n}(\widetilde{z})=\overline{\left|\left\langle\Psi_{n}(\widetilde{z}) \mid \Psi_{n}(0)\right\rangle\right|^{2}}$ for selected states in the chaotic regimes indicated (symbols), and one for $2^{+}$states in a regular region (dashes). The solid curves are our universal predictions 18. As there are no universal predictions in the regular regimes, the dashed curve is only representative.

Figure 9. Distributions of off-diagonal matrix elements $P_{k}\left(U_{n m}(\widetilde{z})\right)$ (where $k=|n-m|$ ). The IBM results for chaotic $J^{\pi}=10^{+}$states (boxes) agree well with the random matrix 
predictions (solid) using $N=50$, as well as with a simple analytic function (dashes). In addition, we show the asymptotic results $P_{k}^{\prime}(\widetilde{z})$ (dots) and $P_{k}^{\prime \prime}(\widetilde{z})$ (dot-dashed) of Ref. [21] which converge for low $k$ to the exact results. For reference, a similar calculation in the regular region for $10^{+}$states is included (crosses).

Figure 10. Distributions of diagonal matrix elements $P_{\widetilde{z}}\left(U_{n n}(\widetilde{z})\right)$ at several values of $\widetilde{z}$ for $10^{+}$state (solid) and the random matrix predictions (dashed). The distribution shifts from a delta function centered at one at $\widetilde{z}=0$, to an asymptotic, non-universal Porter-Thomas distribution for $\widetilde{z} \gg 1$

Figure 11. Distributions of off-diagonal matrix elements $P_{\widetilde{z}}\left(U_{n m}(\widetilde{z})\right)$ at several values of $\widetilde{z}$ for $10^{+}$state (histogram). The distribution shifts from a delta function centered at zero at $\widetilde{z}=0$, to an asymptotic, non-universal Porter-Thomas distribution for $\widetilde{z} \gg 1$ (solid).

Figure 12. Effects of Berry's phase on universal distribution functions. The parameter $\widetilde{z}$ undergoes motion on a closed loop, starting and ending at $\widetilde{z}=0$. Starting at the top, the distribution evolves and eventually bifurcates due to the presence of topological phases. Both results $\widetilde{z}=0.5$ are within the universal regime, but the lower figure shows that the path taken to get to the point of interest can be important. At the bottom, the fraction of matrix elements $f$ that change to $U_{n m}=-1$ depends on the area enclosed. The minimum area needed to achieve the maximum fraction of $1 / 2$ scales as $1 / N$, and is hence rapidly realized in large dimensional systems. 